\title{
Strategic Cooperation in Cost Sharing Games
}

\author{
Martin Hoefer*
}

\begin{abstract}
In this paper we consider strategic cost sharing games with so-called arbitrary sharing based on various combinatorial optimization problems, such as vertex and set cover, facility location, and network design problems. We concentrate on the existence and computational complexity of strong equilibria, in which no coalition can improve the cost of each of its members.

Our main result reveals a connection between strong equilibrium in strategic games and the core in traditional coalitional cost sharing games studied in economics. For set cover and facility location games this results in a tight characterization of the existence of strong equilibrium using the integrality gap of suitable linear programming formulations. Furthermore, it allows to derive all existing results for strong equilibria in network design cost sharing games with arbitrary sharing via a unified approach. In addition, we are able to show that in general the strong price of anarchy is always 1 . This should be contrasted with the price of anarchy of $\Theta(n)$ for Nash equilibria. Finally, we indicate that the LP-approach can also be used to compute near-optimal and near-stable approximate strong equilibria.
\end{abstract}

\section{Introduction}

How can a set of self-interested actors share the cost of a joint investment in a fair and stable way? This fundamental question has motivated a large amount of research in economics in the last decades [40. More recently, this question has been studied in computer science to understand the development of the Internet and questions arising in e-commerce [28. A classic framework to study cost sharing problems without centralized control are cost sharing games, in which cost can be specified as an abstract parameter for each player and/or each coalition. Relevant to real-world optimization problems are cost sharing games, where the cost is tied to the investment into specific resources. Such games based on combinatorial optimization problems have a long tradition in economics. They are usually formulated as coalitional game, i.e., there is a set of players, and each coalition of players has an associated cost value. This value comes from an optimal solution to an underlying optimization problem for the coalition. For example, consider a multicast network design game, in which players strive to establish connections to a common source vertex $s$. This scenario can be formulated as a MST game, in which each vertex $v \neq s$ is a player, and the edges have costs. The cost of a coalition in this game is the cost of the MST for the set $C \cup s$. In the literature many interesting and important coalitional cost sharing games have been studied, e.g., based on problems like MST and Steiner tree [10,16, 21, 22, 32, 37, covering and packing problems [14, facility location [18, 38, or TSP [17. The problem in these games is to devise a stable and fair way of sharing the cost among the players. Coalitional cost sharing games are usually transferable utility (TU) games, i.e., the cost can be shared arbitrarily between the players. This allows for the largest level of generality for possible interactions in the bargaining and coalition formation process. The foremost concept of stability and fairness in TU cost sharing games is the core. The core is a set of imputations, i.e., of distributions of the cost for the complete player set to the players. To be in the core an imputation has to fulfill the additional property that no coalition of players in sum pays more than its associated cost value. Results about the non-emptiness of the core and characterizations of core solutions have been obtained for many of the games mentioned above.

\footnotetext{
${ }^{*}$ Dept. of Computer Science, RWTH Aachen University, Germany, mhoefer@cs.rwth-aachen.de. Supported by DFG through
} UMIC Research Centre at RWTH Aachen University. 
A problem with coalitional cost sharing games is that cost shares represent a strong abstraction from the underlying optimization problem. Players are assumed to contribute on a global level, and the game does not take into account who pays how much for which resource. Such information, however, is crucial when studying the incentives of players in large unregulated settings such as the Internet. The need to understand cost sharing on a strategic level prompted computer scientists to study strategic cost sharing scenarios. On the one hand, there are a number of recent works on designing strategic cost sharing games to obtain favorable Nash equilibrium properties [13. In these games the underlying assumption is that a central authority designs and maintains the solution and dictates cost shares for each player. This is close to assumptions that are made in the area of cost sharing mechanisms, which have received a lot of attention [27, 29, 30, 34. Designing cost shares, e.g., using Shapley value cost sharing [1,6, 15, 31, can yield favorable properties on the existence and cost of Nash equilibria. In contrast, such a model is unsuitable when there is very little control over players and their bargaining options. A model that allows for general cost sharing between players is sometimes referred to as arbitrary cost sharing, and it has been studied in [4, 5, 7, 12, 15, 23, 26]. In these cost sharing games the strategy of a player is a payment function that specifies his exact contribution to the cost of each resource. The outcomes of such strategic cost sharing games based on combinatorial optimization problems will be the subject of this paper.

When studying the outcomes of the interaction of rational agents in strategic games, we need to discuss the appropriate solution concept. The most prominent stability concept in strategic games is the Nash equilibrium (NE). While a NE (in mixed strategies) always exists, a drawback is that it is only resilient to unilateral deviations. In many reasonable scenarios agents might be able to coordinate their actions, and under these circumstances a NE is not a reasonable solution concept. To address this issue we consider the strong equilibrium (SE) in this paper. A strong equilibrium 9 is a state, from which no coalition (of arbitrary size) has a deviation that lowers the cost of every member of the coalition. This resilience to coalitional deviations is highly attractive. On the downside, strong equilibria might not exist in a game. This may be the reason they have not received an equivalent amount of research interest despite their attractive properties. We partly circumvent this problem by studying approximate versions of the strong equilibrium, which is guaranteed to exist. However, our treatment of these aspects is brief and mostly left for future work.

Our main interest is to characterize the existence, social cost, and computational complexity of SE in strategic cost sharing games based on combinatorial optimization problems. Our first insight in Section 2 reveals that the concept of $\mathrm{SE}$ in strategic cost sharing games is equivalent to seemingly stronger notions of super-strong or sum-strong equilibria. Additionally, we show that a SE in a strategic game can always be turned into a core imputation of the corresponding coalitional game defined on the same instance of the optimization problem. Hence, a SE represents a strategic refinement of a core solution, and existence of a SE implies non-emptiness of the core. It also implies that the strong price of anarchy [2] is 1, i.e., in every $\mathrm{SE}$ a solution is bought that is a social optimum to the underyling optimization problem.

In Section 3 we consider a variety of games based on vertex and set cover and various facility location problems. For these games we show an equivalence result of core and SE. In particular, whenever the core in the coalitional game is nonempty, there is a SE for the strategic game. Our main proof technique relies on a connection via linear programming to Owen's linear production model [33, which is one of the most common ways to show non-emptiness of the core in coalitional games [14. Using this machinery we are able to tightly characterize the existence and cost of SE in all these games. Our results extend to special classes of network design problems. This includes, e.g., MST and classes of Steiner Network Design Games [7, 25]. As a byproduct our general approach yields simple proofs for all known results for SE in strategic cost sharing games with arbitrary sharing, which were previously shown [15] via complicated combinatorial arguments.

The equivalence between SE and core solutions is an interesting and notable fact. However, in other cases such as cooperative games with non-transferable utility (NTU games) and appropriate extensions to strategic games a similar equivalence is obvious. Thus, it may be more surprising to observe that the relation between $\mathrm{SE}$ and core solutions in cost sharing games can be quite complicated. In particular, in Section 4 we explore equivalence without relying on linear programming. While in some cases like Terminal Backup Games [4] we can resort to combinatorial arguments, in other interesting games our results are mostly negative. In 
particular, we show that in Steiner Tree connection games or network cutting games equivalence does not hold, i.e., the core might be non-empty but a SE is absent. A similar result is established in Section 5.1 even for simple vertex cover games when we allow resources to be purchased fractionally or in mulitple units. Characterizing SE in these games remains as an intriguing open problem. We observe in Section 5.2 that linear programming can be used to obtain approximate $(\alpha, \beta)$-SE in vertex and set cover, as well as factility location games. Finally, we conclude in Section 6 with some interesting questions for further research.

Our main conceptual contribution is to reveal a non-trivial and close relation between coalitional and strategic games defined on the same instance of the optimization problem. The strategic game can be seen as a strategic variant of the coalitional game. In particular, the cost value of a coalition is the minimax cost level that the coalition can achieve in the strategic game. In addition, our analysis reveals that in many games SE can act as a strategic refinement of rather coarse core solutions. We believe that this inherent connection between traditional coalitional games studied in economics and strategic cost sharing games recently formulated in computer science is of independent interest and should stimulate further research on cost sharing with rational agents.

\subsection{Preliminaries}

We consider classes of cost sharing games based on combinatorial optimization problems. In each of these games there is a set $R$ of resources. Resource $r \in R$ can be bought if the associated cost $c(r) \geq 0$ is paid for. For $R^{\prime} \subseteq R$ let $c\left(R^{\prime}\right)=\sum_{r \in R^{\prime}} c(r)$. We assume that there is set of players $K$. Each player $k \in K$ strives to satisfy a certain constraint on the bought resources. For example, in the case of the set cover problem the player set is the element set $K=E$. The resources are sets $R=\mathcal{S} \subseteq 2^{E}$ over $E$. The constraint of player $e$ states that there must be at least one bought set $S$ with $e \in S$. In a similar way we can base our construction on various cost minimization problems like facility location or network design. We will describe them in more detail in the corresponding sections. However, a common assumption in our problems is a free disposal property, i.e., if for a set of bought resources all player constraints are satisfied, then a superset of bought resources can never make a player constraint become violated.

For a given set of players, resources, and constraints we define two games - a coalitional and a strategic cost sharing game. The coalitional game $\Delta=(K, c)$ is given by the set of players $K$ and a cost function $c: 2^{K} \rightarrow \mathbb{R}_{0}^{+}$that specifies a cost value for every subset of players. For a coalition $C \subseteq K$, the cost is $c(C)=\sum_{r \in R(C)^{*}} c(r)$ for an optimum solution $R(C)^{*} \subseteq R$ for $C$. In particular, $R(C)^{*}$ is a minimum cost set of resources that must be bought to satisfy all constraints of players in $C$. For example, in a set cover game $R(C)^{*}$ is the minimum cost set cover for the elements in $C$. We denote the special case $R^{*}=R(K)^{*}$ as the social optimum.

The goal in a coalitional game is to find a cost sharing of $c(K)$ for the so-called grand coalition $K$. A vector of cost shares $\gamma_{1}, \ldots, \gamma_{k}$ is called an imputation if $\sum_{i \in K} \gamma_{i}=c(K)$. The game $\Delta$ is a transferable utility (TU) game, i.e., we are free to choose $0 \leq \gamma_{i} \leq c(K)$. The central concept of stability and fairness in coalitional games is the core. The core is the set of imputations $\gamma$, for which $c(C) \geq \sum_{i \in C} \gamma_{i}$. Intuitively, when sharing the cost according to a member of the core, no subset of players has an incentive to deviate from the grand coalition and make a separate investment - depending on the underlying optimization problem, e.g., purchase different sets or construct an independent network.

The strategic game $\Gamma=\left(K,\left(S_{i}\right)_{i \in K},\left(c_{i}\right)_{i \in K}\right)$ is specified by strategies and individual cost for each player. The strategy space $S_{i}$ of player $i \in K$ consists of all functions $s_{i}: R \rightarrow \mathbb{R}_{0}^{+}$. Strategy $s_{i}$ allows him to specify for each resource $r \in R$ how much he is willing to contribute to $r$. A resource $r$ is bought if $\sum_{i \in K} s_{i}(r) \geq c(r)$. A vector of strategies $s$ is a state of the game. For a state $s$ we define $\left|s_{i}\right|=\sum_{r \in R} s_{i}(r)$ and the individual cost of player $i$ as $c_{i}(s)=\left|s_{i}\right|$ if the bought resources satisfy his constraint. Otherwise, $c_{i}(s)=\infty$ or a different value that is prohibitively large.

The foremost concept of stability in strategic games is the Nash equilibrium, a state in which no player unilaterally has an incentive to deviate. In this paper, however, we consider coalitional incentives and thus resort to a strengthened version called strong equilibrium [9. A state $s$ has a violating coalition $C \subseteq K$ if there are strategies $s_{C}^{\prime}=\left(s_{i}^{\prime}\right)_{i \in C}$ such that $c_{i}\left(s_{C}^{\prime}, s_{-C}\right)<c_{i}(s)$ for each $i \in C$. A violating coalition has a deviation, in which all players in $C$ strictly pay less. A strong equilibrium is a state $s$ that has no violating 
coalition. Note that in a SE a set of resources is bought such that all player constraints are satisfied. Each resource $r$ is either paid for exactly or not contributed to at all. Thus, a SE represents a cost sharing of a feasible solution for the grand coalition. In addition, we briefly consider the concept of a $(\alpha, \beta)$-SE. These are strategy profiles, which constitute an approximate solution concept. In a $(\alpha, \beta)$-SE no coalition of players can reduce the cost of every member by strictly more than a factor of $\alpha$, and the cost of the bought solution represents a $\beta$-approximation to $c(K)$.

\section{Strong Equilibria and the Core}

Consider a given set of resources $R$ with $\operatorname{costs} c(r)$ and a set of players $K$ with constraints. Our first result reveals that several coalitional equilibrium concepts coincide in strategic games $\Gamma$. In particular, we consider super-strong and sum-strong equilibria. A state $s$ has a weakly violating coalition $C \subseteq K$ if there are strategies $s_{C}^{\prime}=\left(s_{i}^{\prime}\right)_{i \in C}$ such that $c_{i}\left(s_{C}^{\prime}, s_{-C}\right) \leq c_{i}(s)$ for each $i \in C$ and $c_{i^{\prime}}\left(s^{\prime}, s_{-C}\right)<c_{i^{\prime}}(s)$ for at least one $i^{\prime} \in C$. A state $s$ has a sum violating coalition $C \subseteq K$ if there are strategies $s_{C}^{\prime}=\left(s_{i}^{\prime}\right)_{i \in C}$ such that $\sum_{i \in C} c_{i}\left(s_{C}^{\prime}, s_{-C}\right)<\sum_{i \in C} c_{i}(s)$. A super-strong (sum-strong) equilibrium is a state $s$ that has no weakly (sum) violating coalition. Note that every violating coalition is also weakly violating, and every weakly violating coalition is also sum violating. Hence, every sum-strong equilibrium is super-strong, and every super-strong equilibrium is strong. We note this simple fact because we actually show the absence of sum violating coalitions in our proofs below. In general strategic games it is easy to see that the inclusions are strict, i.e., a strong equilibrium might not be sum-strong. In our strategic cost sharing games $\Gamma$, however, every strong equilibrium is sum-strong.

Proposition 1. Every strong equilibrium in a strategic game $\Gamma$ is a sum-strong equilibrium.

Proof. We will show that if a state has a sum violating coalition, it also has a violating coalition. Suppose in a state $s$ there is a sum violating coalition $C$ and a corresponding deviation $s_{C}^{\prime}$. Note that this directly implies $\sum_{i \in C} c_{i}(s)>0$ and $c_{i}\left(s_{C}^{\prime}, s_{-C}\right)=\left|s_{i}^{\prime}\right|$ for all $i \in C$. If a player $i \in C$ has prohibitively large cost in $s$, he has a unilateral deviation purchasing all resources in $R^{*}$ by himself, which obviously yields a violating (singleton) coalition. Hence, we also assume that for all players $i \in C$ we have finite $\operatorname{cost} c_{i}(s)=\left|s_{i}\right|$ in $s$.

First consider players $i \in C$, for which $\left|s_{i}\right|=0$, and denote the set of these players by $C_{0}$. We can construct a state with $\left|s_{i}^{\prime \prime}\right|=0$ for $i \in C_{0}$ as follows. We define $s^{\prime}(r)=\sum_{i \in C} s_{i}^{\prime}(r)$ and let each player pay $s_{i}^{\prime \prime}(r)=\left(\left|s_{i}\right| / \sum_{i \in C}\left|s_{i}\right|\right) \cdot s^{\prime}(r)$. This yields $\left|s_{i}^{\prime \prime}\right|=0$ if and only if $\left|s_{i}\right|=0$. have

Now we define the coalition $C_{1}=C-C_{0}$ and the deviation $s_{C_{1}}^{\prime \prime}=\left(s_{i}^{\prime \prime}\right)_{i \in C_{1}}$. For every player $i \in C_{1}$ we

$$
\left|s_{i}^{\prime \prime}\right|=\sum_{r \in R} \frac{\left|s_{i}\right|}{\sum_{i \in C}\left|s_{i}\right|} \cdot s^{\prime}(r)=\left|s_{i}\right| \cdot \frac{\sum_{i \in C_{0}}\left|s_{i}^{\prime}\right|}{\sum_{i \in C}\left|s_{k}\right|}=\left|s_{i}\right| \cdot \frac{\sum_{i \in C} c_{i}\left(s_{C}^{\prime}, s_{-C}\right)}{\sum_{i \in C} c_{i}(s)}<\left|s_{i}\right|,
$$

using the assumption for $s_{C}^{\prime}$. Note that for each resource $r$ we have same total contribution in $s_{C}^{\prime}$ and $s_{C_{1}}^{\prime \prime}$, hence for every $i \in C_{1}$ we have $c_{i}\left(s_{C_{1}}^{\prime \prime}, s_{-C_{1}}\right)=\left|s_{i}^{\prime \prime}\right|<\left|s_{i}\right|=c_{i}(s)$. This implies that $C_{1}$ is a violating coalition.

We note on the side that for every state $s$, coalition $C$, deviation $s_{C}^{\prime}=\left(s_{i}^{\prime}\right)_{i \in C}$, and finite $\alpha \geq 1$ with $\sum_{i \in C} c_{i}\left(s_{C}^{\prime}, s_{-C}\right)=\alpha \cdot \sum_{i \in C} c_{i}(s)$ we can find in a similar way $C^{\prime}$ and $s_{C^{\prime}}^{\prime \prime}=\left(s_{i}^{\prime \prime}\right)_{i \in C^{\prime}}$ with $c_{i}\left(s_{C}^{\prime \prime}, s_{-C}^{\prime \prime}\right)=$ $\alpha c_{i}(s)$ for every $i \in C^{\prime}$. Thus, the equivalence of strong and sum-strong equilibria holds also for approximate versions of the concepts, in which players must improve their costs by a factor of strictly more than $\alpha$.

We continue to show a general connection between core imputations for the coalitional game $\Delta$ and SE of the strategic game $\Gamma$. We first observe that in a SE players always share the cost of a social optimum $R^{*}$.

Theorem 1. In every strong equilibrium of a strategic game $\Gamma$ the players share the cost of a social optimum. The strong price of anarchy is 1.

Proof. Consider a SE $s$ and the set $R^{\prime}$ of bought resources. Assume for contradiction $c\left(R^{\prime}\right)>c\left(R^{*}\right)$. If all players jointly deviate to purchase $R^{*}$, each player must pay only a fraction of $c\left(R^{*}\right) / c\left(R^{\prime}\right)<1$ of $\left|s_{k}\right|$. A 
player $k$ chooses to pay $s_{k}^{\prime}(r)=c(r) \cdot \frac{\left|s_{k}\right|}{c\left(R^{\prime}\right)}$. If all players jointly deviate to $s^{\prime}$, this obviously strictly decreases the payment of all players. Hence, if $c\left(R^{\prime}\right)>c\left(R^{*}\right)$, then $K$ is a violating coalition for $s$, a contradiction.

Proposition 2. If the strategic game $\Gamma$ has a strong equilibrium, then the coalitional game $\Delta$ has a core solution.

Proof. Consider a SE $s$ of $\Gamma$, which by Theorem 1 is a cost sharing of $R^{*}$, and a coalition $C$. The coalition has the possibility to deviate and contribute just to buy $R(C)^{*}$. In this case it has to share for each resource $r \in R(C)^{*}$ at most the remaining cost on top of the contribution of players in $K \backslash C$, i.e., $c_{C}(r)=$ $c(r)-\sum_{k \in K \backslash C} s_{k}(r)$. If $c_{C}\left(R(C)^{*}\right)<\sum_{k \in C}\left|s_{k}\right|$, the coalition can deviate to $s_{k}^{\prime}(r)=c_{C}(r) \cdot \frac{\left|s_{k}\right|}{c\left(R^{*}\right)}$ for every $k \in C$ and every $r \in R(C)^{*}$, which would represent an improvement for every player in $C$. However, as $s$ is a SE, $C$ must not be violating, and so $c_{C}\left(R(C)^{*}\right) \geq \sum_{k \in C}\left|s_{k}\right|$. Trivially, $c(r) \geq c_{C}(r)$, and so $c\left(R(C)^{*}\right) \geq \sum_{k \in C}\left|s_{k}\right|$. Thus, $\gamma$ with $\gamma_{k}=\left|s_{k}\right|$ is in the core of $\Delta$.

These two simple insights show that non-emptiness of the core is a necessary condition for existence of a SE. In the following we consider various classes of games, in which it is also sufficient. In these cases the SE is a strategic refinement of the core, as it allows to specify a strategic allocation of payments to resources.

\section{Strong Equilibria using Linear Programming}

\subsection{Vertex and Set Cover Games}

In a variety of fundmental games non-emptiness of the core and existence of SE are equivalent. We can relate $\mathrm{SE}$ existence to the core via linear programming duality. For simplicity we outline the general argument in the setting of set cover games. In a set cover game, we are given a set of players as elements $E$ and a set system $\mathcal{S} \subseteq 2^{E}$, where each $S \in \mathcal{S}$ has a cost $c(S) \geq 0$. The constraint of player $e$ is that at least one set $S$ with $e \in S$ must be bought.

Theorem 2. If a set cover game $\Delta$ has a non-empty core, then the strategic game $\Gamma$ has a strong equilibrium.

Proof. We consider the integer programming formulation of set cover. In particular, we consider the following linear relaxation, which employs $x_{S} \geq 0$ instead of $x_{S} \in\{0,1\}$ and thus allows sets to be included fractionally in the solution.

$$
\begin{array}{ll}
\text { Min } & \sum_{S \in \mathcal{S}} x_{S} c(S) \\
\text { subject to } & \sum_{\substack{S: e \in S \\
x_{S} \geq 0}} x_{S} \geq 1 \quad \forall e \in E \\
& \forall S \in \mathcal{S} .
\end{array}
$$

We also consider the corresponding LP dual.

$$
\begin{array}{lll}
\operatorname{Max} & \sum_{e \in E} \gamma_{e} & \\
\text { subject to } & \sum_{e \in S} \gamma_{e} \leq c(S) \quad \forall S \in \mathcal{S} \\
& \gamma_{e} \geq 0 & \forall e \in E .
\end{array}
$$

It has been shown by Deng et al. 14] that the core of $\Delta$ is non-empty if and only if the integrality gap of the underlying set cover problem is 1, i.e., if the LP has an integral optimal solution. With Proposition 2 this is a prerequisite for existence of a SE in $\Gamma$. We strengthen this result by showing that core solutions can also be turned into an allocation of payments to resources for a SE in $\Gamma$. Thus, an integral optimum is also sufficient. 
For the above programs consider the optimum primal solution $x^{*}$ and the optimum dual solution $\gamma^{*}$, where $x^{*}$ is integral and defines a feasible cover. Both $x^{*}$ for the primal and $\gamma^{*}$ for the dual yield the same objective value. Now assign each player $e$ to pay $s_{e}(S)=\gamma_{e}^{*} x_{S}^{*}$. The theorem follows if every set in the cover is purchased exactly and no coalition $C$ can reduce their total payments $\sum_{e \in C}\left|s_{e}\right|$. The first condition is clearly necessary for a SE, the second one implies that no coalition can be sum violating (and thus violating). We first show that the sets are exactly paid for. If $x_{S}^{*}>0$, then due to complementary slackness the inequality $\sum_{e \in E} \gamma_{e}^{*} \leq c(S)$ is tight, hence by this assignment all the purchased sets get exactly paid for.

We now show that no coaltion can reduce the total payments. Suppose a coalition $C$ is violating. We consider an adjusted game derived by iteratively removing elements and payments of other players $e \notin C$. Upon removing an element $e$, we remove its contribution from the costs of sets $S$ including $e$. This yields the cost function $c_{C}(S)$ with

$$
c_{C}(S)=c(S)-\sum_{e \notin C, e \in S} \gamma_{e}^{*} x_{S}^{*} .
$$

It captures the reduced problem of finding a minimum cost cover for coalition $C$ with costs adjuted by the payments of other players $e \notin C$. Note that for this reduced problem the solution $x^{*}$ is still feasible. By obtaining the dual we can set the covering requirement to 0 for every removed element $e \notin C$. Then $\gamma^{*}$ still represents a feasible solution to the LP-dual of the reduced problem. It yields the same objective value as $x^{*}$ for the primal. By strong duality both $x^{*}$ and $\gamma^{*}$ must be optimal solutions to the reduced primal and dual problems. This proves that the total payments of $C$ are optimal. Hence, $C$ cannot be sum violating and not violating, a contradiction. This proves that $s$ is a SE.

The main idea of the proof is to use duality arguments for a cost reduction of resources. In particular, for an optimum $x^{*}$, the objective function can be represented by a linear combination of tight constraints. The multipliers are the optimal dual variables $\gamma^{*}$. This can be used to reduce the costs and show optimality of $x^{*}$ even for every coalition.

For the special case of vertex cover games we can use results from [14 to efficiently compute SE. In particular, a game allows a core solution (and thus a SE) if and only if a maximum matching in the graph has the same size as the minimum vertex cover. This condition can be checked in polynomial time by computing corresponding vertex covers and matchings [14, Theorem 7 and Corollary 7]. Hence, we can check in polynomial time whether a SE exists. If it exists, we can use the computed vertex cover as primal solution for our LP and compute cost shares for a strong equilibrium with the corresponding dual solution.

Corollary 1. In a vertex cover game we can decide in polynomial time if a strong equilibrium exists. If it exists, we can compute a strong equilibrium in polynomial time.

In addition, we can check in polynomial time whether a given strategy profile is a SE.

Corollary 2. Given a state $s$ for a vertex cover game $\Gamma$ we can verify in polynomial time if it is a strong equilibrium.

If the strategy profile is a SE, it must exactly pay for a vertex cover of the problem. This yields a primal solution for the LP. In addition, the accumulated cost shares of players must yield a corresponding dual solution. Finally, both primal and dual solutions must generate the same value of the objective function. This is a sufficient and necessary condition for being a SE, which can be checked in polynomial time.

Another interesting case are edge cover games. Here players are the vertices of a graph and resources are the edges. Each vertex wants to ensure that at least one incident edge is bought. Using the characterization of the non-emptiness of the core in [14, Theorem 8 and Corollary 8] we can obtain similar results for this game as well.

Corollary 3. In an edge cover game $\Gamma$ we can decide in polynomial time if a strong equilibrium exists. If it exists, we can compute a strong equilibrium in polynomial time. Given a state $s$ for an edge cover game $\Gamma$ we can verify in polynomial time if it is a strong equilibrium. 


\subsection{Facility Location Games}

Another class of games that can be handled via similar arguments are facility location games. We outline the arguments on the simple class of uncapacitated facility location games (UFL games) and show below how to extend this approach to a more general class of games considered in 18,23. In a UFL problem there is a set $T$ of terminals and a set $F$ of facilities. We set $n_{t}=|T|$ and $n_{f}=|F|$. Each facility $f \in F$ has an opening $\operatorname{cost} c(f) \geq 0$, for each terminal $t \in T$ and each facility $f \in F$ there is a connection cost $c(t, f) \geq 0$. The goal is to open a subset of facilities and buy a set of connections of minimum total cost, such that each terminal is connected to an opened facility. In the UFL game each player owns a terminal, i.e., $K=T$. The constraint of player $t$ is satisfied if there is a bought connection $(t, f)$ to some opened facility $f$. We can formalize the UFL problem by an integer program as follows:

$$
\begin{array}{lll}
\text { Min } & \sum_{f \in F} c(f) y_{f}+\sum_{t \in T} c(t, f) x_{t f} \\
\text { subject to } & \sum_{f \in F} x_{t f} \geq 1 & \text { for all } t \in T \\
& y_{f}-x_{t f} \geq 0 & \text { for all } t \in T, f \in F \\
& y_{f}, x_{t f} \in\{0,1\} & \text { for all } t \in T, f \in F,
\end{array}
$$

Theorem 3. If a UFL game $\Delta$ has a non-empty core, then the strategic game $\Gamma$ has a strong equilibrium.

Proof. We again use the linear relaxation, which can be obtained by replacing $y_{f}, x_{t f} \in\{0,1\}$ by $y_{f}, x_{t f} \geq 0$. Then the dual can be given by

$$
\begin{array}{lll}
\operatorname{Max} & \sum_{t \in T} \gamma_{t} & \\
\text { subject to } & \sum_{t \in T} \gamma_{t}-\delta_{t f} \leq c(t, f) & \text { for all } t \in T, f \in F \\
& \sum_{t \in T} \delta_{t f} \leq c(f) & \text { for all } f \in F .
\end{array}
$$

It has been shown by Goemans and Skutella [18] that the core of $\Delta$ is non-empty if and only if the integrality gap of this LP is 1 . We can now argue similarly as before. An integral optimum solution $\left(x^{*}, y^{*}\right)$ to the LP-relaxation represents a partition of the terminal set $T$ into a collection of stars, one for each facility $f$. The constraints corresponding to these sets hold with tightness, and we can assign each player $t$ to pay for her terminal the amount $s_{t}(t, f)=\left(\gamma_{t}^{*}-\delta_{t f}^{*}\right) x_{t f}^{*}$ as connection cost to $f$, in which $\left(\gamma^{*}, \delta^{*}\right)$ is the optimum solution to the dual. For the opening costs $s_{t}(f)=\delta_{t f}^{*} y_{f}^{*}$. In total this pays exactly for all costs of the solution by duality.

Suppose there is a violating coalition $C$. We again remove players in $K-C$ and reduce the costs of connections and facilities by the respective contributions. In order to represent a violating coalition, the players in $C$ must be able to deviate and reduce their total sum of payments. However, the solution $\left(x^{*}, y^{*}\right)$ has the same value for the reduced LP of coalition $C$ as $\left(\gamma^{*}, \delta^{*}\right)$ for the dual of the reduced LP. By duality both solutions remain optimal. Thus, coalition $C$ is purchasing an optimal solution against the payments of players in $K-C$ and has no possibility to reduce the total payments. This is a contradiction to $C$ being a violating coalition.

This result can be combined with insights from [18] to characterize computational properties of SE. In particular, we can decide in polynomial time if a given strategy profile for $\Gamma$ is a SE. We first check if the payments of players are made only to their own connection and opening costs. Then we accumulate contributions to cost shares and check if this yields a core solution - i.e., if the primal solution (given by the purchased solution to the facility location problem) and the dual solution (given by the cost shares) correspond to each other and yield the same optimal value for primal and dual LPs.

Corollary 4. Given a strategy vector for a UFL game $\Gamma$ we can verify in polynomial time if it is a strong equilibrium. 
As verfication is in $\mathrm{P}$, the problem of computing a strong equilibrium is in NP. In fact, in [18 it is shown for a class of UFL games that deciding the existence of a core solution is NP-complete. As existence of SE and core solutions is equivalent, this yields the following result.

Corollary 5. It is NP-complete to decide if a given UFL game $\Gamma$ has a strong equilibrium.

In the Appendix we show how to extend this result to connection-restricted facility location games (CRFL games) from [18, in which access to a facility $f$ can be obtained only by certain allowed coalitions $\mathcal{A}_{f} \subseteq 2^{T}$. We consider the special case of closed games (CCRFL games), in which the set system $\mathcal{A}_{f}$ of allowed coalitions is downward closed, i.e., each subset of an allowed coalition is also an allowed coalition. This simplifies the specific allocation of the cost shares to connections and facilities. While the closed property is a restriction, we note that many variants of facility location arising in practice fall into this class of games, e.g., problems with capacity or incompatibility constraints. We believe that equivalence between core and SE also holds for CRFL games in full generality, but a proof of this statement remains as an open problem.

Theorem 4. If a CCRFL game $\Delta$ has a non-empty core, then the strategic game $\Gamma$ has a strong equilibrium. Given a strategy vector for a CCRFL game $\Gamma$ we can verify in polynomial time if it is a strong equilibrium.

\subsection{Connection Games}

In this section we use a linear program to formulate network design games in directed and undirected graphs. Perhaps the most frequently studied variant is a connection game originally formulated in [7]. In this game there is a graph $G=(V, E)$, resources are the edges, and each edge has a non-negative cost $c(e) \geq 0$. There is a set of players $K$, and each player $k$ has a source-sink pair $\left(s_{k}, t_{k}\right)$. A player is satisfied if there is a path of bought edges connecting his source and sink. This is a game based on the Steiner network problem in graphs [19. In a variant based on Steiner Tree called the single source game, every player has the same source $s$. Here we characterize existence of SE based on a Flow-LP previously studied, e.g., in [37,39.

Theorem 5. If the Flow-LP has an integral optimum solution, then the strategic connection game $\Gamma$ has a strong equilibrium.

Proof. We formulate the mixed integer program for the problem in directed graphs. It is simple to adjust it to undirected graphs, where we use only one variable $y_{i j}$ for each (undirected) edge $e=(i, j) \in E$.

$$
\begin{array}{ll}
\text { Min } & \sum_{(i, j) \in E} c_{i j} y_{i j} \\
\text { s.t. } & \sum_{\{j \mid(i, j) \in E\}} f_{i j}^{k}-\sum_{\{i \mid} f_{j i}^{k} \geq 1 \quad \text { for } i=s_{k} \\
& \sum_{\{j \mid(i, j) \in E\}} f_{i j}^{k}-\sum_{\{i \mid} f_{j i}^{k} \geq 0 \quad \text { for } i \neq s_{k}, t_{k} \\
& y_{i j}-f_{i j}^{k} \geq 0 \quad \text { for }(i, j) \in E, k \in K \\
& f_{i j}^{k} \geq 0, y_{i j} \in\{0,1\} \quad \text { for }(i, j) \in E, k \in K
\end{array}
$$

In this MIP we optimize for each player $k$ a flow, which is required to have value 1 by the constraints at the source, and which can only exit through the sink. The individual flows are coordinated by capacity constraints $y_{i j}-f_{i j}^{k} \geq 0$. Each edge that is used by at least one player fractionally has to be fully paid for in the objective function. We can relax this program by using $y_{i j} \geq 0$. Then the dual can be formulated using variables $\delta_{i}^{k}$ for the flow conservation constraints and $\gamma_{i j}^{k}$ for the coordination constraints. Intuitively, the values $\delta_{i}^{k}$ introduce a node potential of contributions, and $\gamma_{i j}^{k}$ can be seen as contributions towards the edges that are bought.

It has been observed in [37] that this is program is wihtin Owens linear production model. Hence, if the integrality gap is 1 , the optimal dual solution yields a core solution. Using similar arguments as before, we 
can also show that in this case a SE exists. In particular, each player pays $s_{k}(i, j)=y_{i j}^{*}\left(\gamma_{i j}^{k *}\right)$ towards edge $(i, j)$. For a coalition $C$ we can again reduce costs of edges by removing players of $K-C$. Due to the additive structure of the LP, the primal and dual optimal solutions remain optimal for the reduced LPs. This means no coalition can reduce total payments, and no coalition can be violating. This proves the theorem.

This insight allows us to derive one of the main results shown in [15] in a simple and compact way.

Theorem 6. [15] For a single source connection game $\Gamma$ on a directed series-parallel graph a strong equilibrium always exists and can be computed in polynomial time.

The existence result follows easily by observing that the Flow-LP for single source games on directed series-parallel graphs has integrality gap 1. A proof can be derived from [35. Solving this LP resembles the construction in [15.

Another class, in which the above LP can be used to show existence of SE, are games based on the minimum spanning tree (MST) problem. MST games are single source games, in which in every vertex of $G$ is a sink node for at least one player.

Theorem 7. In every MST game $\Gamma$ there is a strong equilibrium, which can be computed in polynomial time.

For the problem in directed graphs, a SE can be computed from dual solutions of the LP [37. One of these dual solutions is the core solution derived for the original non-emptiness proof [21. In this solution, each player $k$ pays exactly for the unique arc of the tree leaving his sink $t_{k}$. This rule has also been described by Bird [10]. It requires an easy argument to see that it yields a SE, even for the MST game in undirected graphs.

While in these cases we have guaranteed existence and efficient algorithms to compute SE, the problem of deciding the existence of SE is NP-hard. This follows from a simple adjustment, which allows to interpret UFL games as single source connection games on directed graphs.

Corollary 6. It is NP-hard to decide if a given single source connection game $\Gamma$ on a directed graph has a strong equilibrium.

\section{Strong Equilibria beyond Linear Programming}

\subsection{Connection Games}

For set cover and facility location games the integrality gap condition provides a complete characterization of games $\Delta$ having core solutions. With our theorems we obtain a complete characterization also for the existence of SE in strategic games $\Gamma$. For network design games like the connection game, the integrality gap condition is sufficient to show existence of SE and non-emptiness of the core, but it is not necessary. A tight characterization of games with non-empty core has not been obtained so far.

For strategic games and SE it has been shown in [15] that there is a single source connection game without $\mathrm{SE}$, but the corresponding cooperative game to their example does not allow a core solution as well. By Proposition 2, however, this is a prerequisite for SE existence. Coalitional connection games with an empty core (and thus without SE) have already been presented in 22]. We here show that even a spanning property of the optimum solution $R^{*}$ is not sufficient to guarantee $\mathrm{SE}$ existence or to obtain $\mathrm{SE}$ from core solutions. This implies that the relation between core and SE is not as robust as for the other games considered previously. A complete characterization of the existence of SE in (single source) connection games remains as an open problem.

Lemma 1. There are corresponding strategic and coalitional single source connection games $\Gamma$ and $\Delta$ such that $R^{*}$ is a MST of $G$ and $\Delta$ has a core solution but $\Gamma$ has no strong equilibrium.

Proof. Our example game is shown in Fig. 1. It is based on a game presented in [22, which consisted only of the three lower layers up to node $s^{\prime}$. It was shown that this game has an empty core, but $R^{*}$ passes through all vertices of $G$. This also implies that there can be no SE. 

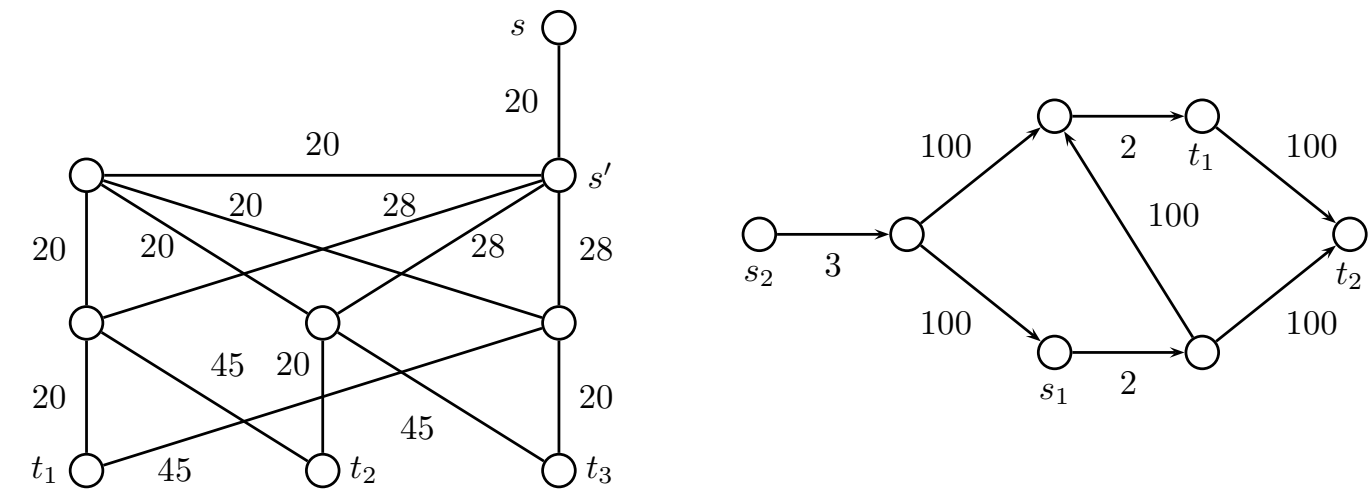

Figure 1: Left: A single source connection game with 3 players, a non-empty core, but without a SE. $R^{*}$ is an MST of $G$ and consists of all edges of cost 20. Right: A multicut game on a directed graph with 2 players and a non-empty core. The game has no NE.

To obtain our game in Fig. 1, we added the new source $s$ and an edge of cost 20 to the old source $s^{\prime}$. Then the constraints for the contributions of the coalitions allow a feasible cost sharing by assigning each player a share of $160 / 3 \approx 53.33$. This removes the incentives to deviate on a global scale, which is sufficient for non-emptiness of the core. On a local scale, however, the instable structure up to $s^{\prime}$ is still intact. The additional contributions towards $\left(s^{\prime}, s\right)$ do not change the strategic incentives within the lower parts of the graph. It can be verified that in this game no SE exists. This proves the lemma.

\subsection{Terminal Backup Games}

In this section we study games based on the terminal backup problem [4,8. In this game there is a graph $G=(V, E)$, each player is a vertex $(K \subset V)$, and resources are the edges with costs $c(e) \geq 0$. Each player strives to be connected to at least $d-1$ other player vertices, for $d \geq 2$. It has been shown in 8 , that the terminal backup problem can be solved in polynomial time for $d=2$. Here we show that every core solution can be turned into a SE for these games. In addition, we show how to decide if a game has a SE and how to obtain $\mathrm{SE}$ in polynomial time if they exist.

Theorem 8. If a terminal backup game $\Delta$ with $d=2$ has a non-empty core, then the strategic game $\Gamma$ has a strong equilibrium.

Proof. Suppose there is a core solution in $\Delta$, but there is no $\mathrm{SE}$ in $\Gamma$. We can allocate the cost shares from the core for each player $k$ to a consecutive subtree starting at their vertex. Furthermore, we can adjust the graph such that $R^{*}$ is only composed path components with two player vertices at the ends, or of star components with at most three player vertices. This adjustment can be achieved by replacing each non-player vertex with a clique of sufficiently many vertices and clique edges of cost 0 .

In a star component, each player pays a cost of at most the connection to the center. For contradiction assume that the cost share of player $i$ in the core solution is $\epsilon>0$ larger than the cost of the connection to the star center. Then every other player in the component must pay at most its' own connection cost to the star center minus $\epsilon$. Thus, either the component is a path, or player $i$ is left to pay at least $2 \epsilon$, which is a contradiction. This shows that there exists an allocation of core cost shares to the edges such that each player pays for a consecutive path starting at his terminal.

For the sake of contradiction assume that this allocation is not a SE. Suppose $C$ is a violating coalition of players. In their improvement the players of $C$ can improve by changing their connections and create a new component. If this new component is paid for fully by the players in $C$, this corresponds to a constraint 
considered for the core solution. Hence, the players in such a new component cannot all profit from such a deviation.

On the other hand, suppose players use edges to create their new component, for which part of the cost is paid for by players not in $C$. These other players pay a consecutive subtree starting at their terminals. Hence, their connection requirement will be satisfied in the new component as well. Thus, they and all the players located in the bought subtree incident to their vertex can be added to the coalition and the deviation. The costs can be redistributed such that everybody improves and pays a lesser amount than before. This again yields a set of improving players that pays completely for their component. However, as such deviations are covered by the core constraints, this is again a contradiction to the cost shares being a core solution. This completes the proof of the theorem.

The above property allows us to efficiently determine if SE and core solutions exist and to compute them in polynomial time if they exist.

Corollary 7. There is a polynomial time algorithm to determine if a coalitional terminal backup game $\Delta$ with $d=2$ has a core solution and if the strategic game $\Gamma$ has a strong equilibrium. If they exist, a core solution and a strong equilibrium can be computed in polynomial time.

Proof. We can compute an optimal solution in polynomial time. We then decide if a core solution exists as follows. The structure of the problem implies that optimal solutions can be assumed as compositions of components for 2 or 3 players. There are only a polynomial number of such coalitions, and the optimum solution for each such coalition can be found in polynomial time for each of them. Hence, the set of inequalities that characterizes the core is only of polynomial size and can be obtained in polynomial time. Thus, we can check in polynomial time if this set of inequalities has a solution and in this way obtain a member of the core. Given a core solution, we can use the computed optimum solution and our structural insight about SE to find the appropriate allocation of payments to edges in polynomial time.

For larger connectivity requirements of $d \geq 4$ we construct games where the consecutive payment condition of Theorem 8 is violated. In this case, a core solution cannot be turned into a SE.

Lemma 2. For any $d \geq 4$ there is a coalitional terminal backup game $\Delta$ with a core solution and a corresponding strategic game $\Gamma$ without a strong equilibrium.

In fact, our example game can be derived directly with the single source connection game in Fig. 1 above. We simply replace the source $s$ by a clique of 4 or more terminals and 0 -cost edges.

\subsection{Network Cutting Games}

In this section we briefly discuss a network cutting game, in which there is a graph $G=(V, E)$ and each player strives to disconnect a subset $S_{k} \subset V$ from another subset $T_{k} \subset V$. Each edge $e \in E$ has a cost $c(e)>0$ for disconnection. This approach yields coalitional and strategic games based on a variety of minimum-cut problems like $s$-t-cut, multicut, mulitway cut, etc. It is introduced and studied with respect to NE in the special cases of mulitway cut and multicut in [3].

More formally, for each player $k$ denote by $\mathcal{P}_{k}$ the set of all paths in $G$ from a node in $S_{i}$ to a node in $T_{i}$. When we introduce a variable $x_{e}$ for each edge $e \in E$, then for each path $P \in \mathcal{P}_{k}$ player $k$ has the

constraint $\sum_{e \in P} x_{e} \geq 1$. Note that these are simple 0/1-covering constraints, and thus the resulting integer program is a special case of the set cover integer program presented above. In particular, we can simply regard paths as elements and edges as sets. This implies that if the integrality gap is 1 , we have existence of core solutions and SE. For instance, this holds on directed and undirected graphs for single-source games that have $S_{i}=\{s\}$ for each $i \in K$.

Theorem 9. If the Covering-LP has an integral optimum solution, then the strategic network cutting game $\Gamma$ has a strong equilibrium. 
Note that there is a subtle twist to this observation. While in the set cover game every element (i.e., every path) is a player, in the cutting game players strive to cover multiple elements (i.e., cut multiple paths). The previous theorem still holds, because by clustering elements we simply reduce the granularity of possible coalitions to those, which can be obtained by the union of sets $\mathcal{P}_{k}$. In fact, by this transformation we increase the set of games that allow a strong equilibrium and a core solution.

Proposition 3. There are network cutting games $\Gamma$ with strong equilibria, for which the underlying network cutting problem has an integrality gap of more than 1.

Proof. Consider a network multiway cut game, in which every player $i$ has a vertex $s_{i} \in V$ and wants to disconnect it from every other player vertex, i.e., $T_{i}=\left\{s_{j}: i \neq j \in K\right\}$. Consider a star, in which the player vertices are exactly the leaves and all edges have cost 1 . This class of instances is known to have the maximum integrality gap of $2-1 / k$ for the covering LP of the network multicut problem. In particular, the fractional optimum solution assigns each edge to be in the cut with $x_{e}=1 / 2$, while the integral optimum fully cuts all but one edge. In a SE we pick one player $i$ to be uncut. Each other player $j \neq i$ is assigned to purchase the edge incident to $s_{j}$ completely. Note that every coalition $C$ without the uncut player $i$ must pay at least $k$ to remain disconnected from $i$. Every other coalition must pay at least $k-1$. Hence, no coalition can reduce their payments in sum, and the existence of a SE and a core solution follows.

A similar observation can be made for multiway cut games, in which geometric LP relaxations [11] have an integrality gap of more than 1 . In fact, it can be observed that the arguments from [3] for existence of optimal NE can be extended in a straightforward way to show that every mutiway cut game on an undirected graph admits a SE. In general network cutting games, however, the set of strategic games with a SE is not equivalent to the set of cooperative games with a non-empty core.

Lemma 3. There are corresponding coalitional and strategic network cutting games $\Delta$ and $\Gamma$ such that $\Delta$ has a core solution but $\Gamma$ has no strong equilibrium.

Proof. For undirected graphs we consider two players and a star graph. We set $S_{1}=\left\{s_{1}\right\}, S_{2}=\left\{s_{2}\right\}$, $T_{1}=\left\{t_{1}\right\}$ and $T_{2}=\left\{s_{1}, t_{1}\right\}$. The edge costs to the center node $u$ are $c\left(s_{1}, u\right)=c\left(t_{1}, u\right)=2$ and $c\left(s_{2}, u\right)=3$. The set of core solutions is $\gamma_{1}=2-\epsilon$ and $\gamma_{2}=2+\epsilon$ for $0 \leq \epsilon \leq 1$. Note that the unique optimum solution is to cut $\left(s_{1}, u\right)$ and $\left(t_{1}, u\right)$. In such a solution, however, if $\left|s_{1}\right|>0$, player 1 can unilaterally improve by removing the larger of his payments. Player 2 does not pay for both edges, because paying only for $\left(s_{1}, u\right)$ is cheaper.

For directed graphs we can even leave $T_{2}=\left\{t_{2}\right\}$ as a singleton. We transform the graph to the one shown in Fig. 1. A similar argument shows non-existence of SE.

This construction implies that when we relax the assumption that every element or terminal is a player in a set cover or facility location game, equivalence between core and SE does not hold anymore. On another note, the proof shows absence of NE in general strategic network cutting games on undirected games. For directed graphs the absence of NE holds true even for minimum multicut games, in which $S_{i}$ and $T_{i}$ are singleton sets for all players $i \in K$.

\section{$5 \quad$ Extensions}

\subsection{Fractional and Non-Binary Resources}

Apart from equivalence of core and SE, a natural question is to characterize cases when we can derive SE from core solutions using linear programming. This was possible when the integrality gap was 1 in all the cases described above. With exception of the CCRFL games, all games studied above yield linear constraints that fall into one of two classes. One type of constraint is $\sum_{i} x_{i} \geq 1$, i.e., a simple covering constraint with $0 / 1$ coefficients, by which we can express exactly the vertex and set cover games. The other constraint type is $y_{i}-\sum_{j} x_{i j} \geq 0$, i.e., a coordination constraint that requires a resource to become bought when at least 
one player uses it. This second type of constraint allows us to treat facility location and network design cut games. What happens if we slightly generalize these constraints?

As an example let us first consider dropping the integrality requirement. Using results on Owen's linear production model one can show, for instance, that vertex cover games always allow a core solution if vertices and sets can be bought in fractional amounts. Does a SE also exist for strategic games in these cases? To answer this question we must adjust the strategic game to allow vertices to be bought fractionally. The obvious adjustment is to assign a fraction proportional to the total payment. In a state $s$ of the strategic fractional vertex cover game a vertex $v$ is bought to the degree $x_{v}=\sum_{k \in K} s_{k}(e) / c(v)$. For a player $k$ corresponding to edge $e=(u, v)$ the individual cost is $\left|s_{k}\right|$ if $x_{u}+x_{v} \geq 1$ and prohibitively large otherwise.

A second, closely related variant is the case when we keep the integrality condition, but we increase the covering requirements and allow multiple units of a resouce to be bought. In particular, we change the constraints to a type $\sum_{i} x_{i} \geq b$, where $b>0$ and $x_{i} \in \mathbb{N}$. As for the fractional games the total payments of the players determine the number of units bought of a resource. We term these games non-binary vertex cover games. More formally, in a state $s$ we have $x_{u}=\left\lfloor\sum_{k \in K} s_{k}(u)\right\rfloor$. Player $k$ corresponding to edge $(u, v)$ has a required coverage of $b_{k} \in \mathbb{N}$ and individual cost $c_{k}(s)=\left|s_{k}\right|$ if $x_{u}+x_{v} \geq b_{k}$ and prohibitively large otherwise.

Note that for both of these game classes Proposition 2 and Theorem 1 continue to hold. In contrast to our results above, however, we show next that there might be no SE - although non-emptiness of the core can be established via the same linear programming machinery that was used before.

Theorem 10. There are corresponding strategic and coalitional fractional or non-binary vertex cover games $\Delta$ and $\Gamma$ such that $\Delta$ has a core solution but $\Gamma$ has no strong equilibrium.

Proof. For both variants the proof follows with a triangle, vertex $\operatorname{costs} c(u)=3, c(v)=5$, and $c(w)=7$, and players 1 to 3 corresponding to edges $(u, w),(u, v)$ and $(v, w)$, respectively.

In the fractional game the unique optimum solution to the underlying vertex cover problem is $x_{u}^{*}=x_{v}^{*}=$ $x_{w}^{*}=1 / 2$, and the unique core solution is $\gamma_{1}=2.5, \gamma_{2}=0.5$ and $\gamma_{3}=4.5$. Theorem 1 yields that $x^{*}$ has to be purchased in every SE, but no player is willing to contribute to $w$. We obviously must have $s_{2}(w)=0$. If $s_{1}(w)>0$, player 1 can deviate unilaterally and achieve the amount $s_{1}(w) / 7$ of coverage by contribution to $u$ with less payments. The same holds for player 2 and vertex $v$.

For the non-binary version, we set all covering requirements to $b_{1}=b_{2}=b_{3}=4$. Then the unique optimum $x^{*}$ to the underlying vertex cover problem and the unique core payments $\gamma$ are the same as before scaled by factor 4 . Observe that we have an integrality gap of 1 in this game. The core solution is unique, so with Proposition 2 we know that in every SE $\left|s_{1}\right|=10$ and $\left|s_{2}\right|=2$. This implies $4 \leq s_{1}(w) \leq 6$. By removing this payment from $w$, player 1 reduces the number of units bought of $w$ by exactly 1 . However, he can obtain an additional unit of $u$ at a cost of 3 . This yields a profitable unilateral deviation and proves the theorem.

This shows that in the class of non-binary vertex cover games neither non-emptiness of the core nor an integrality gap of 1 can guarantee the existence of SE.

\subsection{Approximate Equilibria}

We have presented a method to derive SE in strategic cost sharing games via linear programming. A disadvantage of the concept of SE is that they might not exist in a game. However, our approach proves to be applicable even to approximate SE. Using primal-dual algorithms we can compute $(\alpha, \beta)$-SE with small (constant) ratios in polynomial time for vertex cover, set cover, and facility location games. The proof for the following theorem can be derived directly from arguments in [12,23].

Theorem 11. There are efficient primal-dual algorithms to compute $(2,2)$-SE for vertex cover, $(f, f)-S E$ for set cover (where $f$ is the maximum frequency of any element in the sets), and (3,3)-SE for metric UFL games in polynomial time. 
We provide a sketch of the proof here. In [12,23, we have observed that the results stated in the theorem hold for $(\alpha, \beta)$-NE with the same ratios. Consider the proof for, e.g., the (2,2)-NE in vertex cover games in [12. The primal-dual algorithm iteratively raises payments made by single edges until one of the incident vertices becomes bought. Players can own multiple edges, but their total payments are made up by the sum of payments assigned to their edges. In fact, the payments assigned by the algorithm are independent of which player owns which edge. A deviation of a player owning multiple edges is equivalent to a coordinated deviation the coalition of single edge players. Thus, the proof that the algorithm computes $(2,2)$-NE shows that the state computed by the algorithm allows no coalitional deviation of single edge players that decreases their payments in sum by a factor of strictly more than 2 . In this case we obviously cannot have a deviation in which every player of a coalition reduces his cost by a factor of strictly more than 2 . These observations yield the result for vertex cover games, and similar arguments can be applied using the algorithms for set cover and facility location games treated in [23].

\section{Conclusions and Open Problems}

In (single source) connection, network cutting and fractional and non-binary games the use of linear programming duality does not necessarily yield a complete characterization of the games that admit SE. In these games and other interesting variants of cost sharing in network design our work opens up numerous interesting research problems regarding the characterization and computation of exact and approximate SE.

More generally, we believe that the linkage between core and strong equilibrium could be present in other cost sharing games, which go beyond the classes of games treated in this paper. Exploring these classes of games is an interesting avenue for further research. More concretely, our games have linear programming formulations that lie within Owens linear production model. Non-emptiness of the core, however, can also be shown within a more general class of problems. This more general framework, termed generalized linear production model in [20, has a non-additive structure, and it encompasses for instance the cut-based LPformulation for Steiner Network problems [36. It is a fascinating open problem to see if this framework can also be used to derive exact and approximate SE in strategic cost sharing games.

\section{Acknowledgement}

The author would like to thank Elliot Anshelevich and Bugra Caskurlu for valuable discussions and feedback about the results in this paper.

\section{References}

[1] Susanne Albers. On the value of coordination in network design. SIAM J. Comput., 38(6):2273-2302, 2009.

[2] Nir Andelman, Michal Feldman, and Yishay Mansour. Strong price of anarchy. Games Econ. Behav., 65(2):289-317, 2009.

[3] Elliot Anshelevich, Bugra Cascurlu, and Ameya Hate. Selfish multiway cut and multicut games. Manuscript, March 2010.

[4] Elliot Anshelevich and Bugra Caskurlu. Exact and approximate equilibria for optimal group network formation. In Proc. 17th European Symposium on Algorithms (ESA), pages 239-250, 2009.

[5] Elliot Anshelevich and Bugra Caskurlu. Price of stability in survivable network design. In Proc. 2nd Intl. Symp. Algorithmic Game Theory (SAGT), pages 208-219, 2009.

[6] Elliot Anshelevich, Anirban Dasgupta, Jon Kleinberg, Tim Roughgarden, Éva Tardos, and Tom Wexler. The price of stability for network design with fair cost allocation. SIAM J. Comput., 38(4):1602-1623, 2008. 
[7] Elliot Anshelevich, Anirban Dasgupta, Éva Tardos, and Tom Wexler. Near-optimal network design with selfish agents. Theory of Computing, 4:77-109, 2008.

[8] Elliot Anshelevich and Adriana Karagiozova. Terminal backup, 3D matching, and covering cubic graphs. In Proc. 39th Symp. Theory of Computing (STOC), pages 391-400, 2007.

[9] Robert Aumann. Acceptable points in general cooperative n-person games. In Contributions to the Theory of Games IV, volume 40 of Annals of Mathematics Study, pages 287-324. 1959.

[10] C. Bird. On cost allocation for a spanning tree: A game theoretic approach. Networks, 6:335-350, 1976.

[11] Gruia Calinescu, Howard Karloff, and Yuval Rabani. An improved approximation algorithm for multiway cut. J. Comput. Syst. Sci., 60(3):564-574, 2000.

[12] Jean Cardinal and Martin Hoefer. Selfish service installation in networks. In Proc. 2nd Intl. Workshop Internet 83 Network Economics (WINE), pages 174-185, 2006.

[13] Ho-Lin Chen, Tim Roughgarden, and Gregory Valiant. Designing network protocols for good equilibria. SIAM J. Comput., 39(5):1799-1832, 2010.

[14] Xiaotie Deng, Toshihide Ibaraki, and Hiroshi Nagamochi. Algorithmic aspects of the core of combinatorial optimization games. Math. Oper. Res., 24(3):751-766, 1999.

[15] Amir Epstein, Michal Feldman, and Yishay Mansour. Strong equilibrium in cost sharing connection games. Games Econ. Behav., 67(1):51-68, 2009.

[16] Ulrich Faigle, Sándor Fekete, Winfried Hochstättler, and Walter Kern. On the complexity of testing membership in the core of min-cost spanning trees. Intl. J. Game Theory, 26:361-366, 1997.

[17] Ulrich Faigle, Sándor Fekete, Winfried Hochstättler, and Walter Kern. On approximately fair cost allocation in Euclidean TSP games. OR Spektrum, 20(1):29-37, 1998.

[18] Michel Goemans and Martin Skutella. Cooperative facility location games. J. Algorithms, 50(2):194$214,2004$.

[19] Michel Goemans and David Williamson. A general approximation technique for constrained forest problems. SIAM J. Comput., 24(2):296-317, 1995.

[20] Daniel Granot. A generalized linear production model: A unifying model. Math. Prog., 34:212-222, 1986.

[21] Daniel Granot and Gur Huberman. On minimum cost spanning tree games. Math. Prog., 21:1-18, 1981.

[22] Daniel Granot and Michael Maschler. Spanning network games. Intl. J. Game Theory, 27:467-500, 1998.

[23] Martin Hoefer. Non-cooperative facility location and covering games. In Proc. 17th Intl. Symp. Algorithms and Computation (ISAAC), pages 369-378, 2006.

[24] Martin Hoefer. Competitive cost sharing with economies of scale. In Proc. 8th Latin American Theoretical Informatics Conference (LATIN), pages 339-349, 2008.

[25] Martin Hoefer. Non-cooperative tree creation. Algorithmica, 53(1):104-131, 2009.

[26] Martin Hoefer and Piotr Krysta. Geometric network design with selfish agents. In Proc. 11th Conf. Computing and Combinatorics (COCOON), pages 167-178, 2005.

[27] Nicole Immorlica, Mohammad Mahdian, and Vahab Mirrokni. Limitations of cross-monotonic cost sharing schemes. ACM Trans. Algorithms, 4(2), 2008. Special Issue SODA 2005. 
[28] Kamal Jain and Mohammad Mahdian. Cost sharing. In Noam Nisan, Éva Tardos, Tim Roughgarden, and Vijay Vazirani, editors, Algorithmic Game Theory. Cambridge University Press, 2007.

[29] Kamal Jain and Vijay Vazirani. Applications of approximation algorithms to cooperative games. In Proc. 33rd Symp. Theory of Computing (STOC), pages 364-372, 2001.

[30] Jochen Könemann, Stefano Leonardi, Guido Schäfer, and Stefan van Zwam. A group-strategyproof cost sharing mechanism for the Steiner forest game. SIAM J. Comput., 37(5):1319-1341, 2008.

[31] Stefano Leonardi and Piotr Sankowsi. Network formation games with local coalitions. In Proc. 26th Symp. Principles of Distributed Computing (PODC), pages 299-305, 2007.

[32] Nimrod Megiddo. Cost allocation for Steiner trees. Networks, pages 1-6, 1978.

[33] Guillermo Owen. On the core of linear production games. Math. Prog., 9:358-370, 1975.

[34] Martin Pál and Éva Tardos. Group strategyproof mechanisms via primal-dual algorithms. In Proc. 44th Symp. Foundations of Computer Science (FOCS), pages 584-593, 2003.

[35] A. Prodon, T.M. Libeling, and H. Gröflin. Steiner's problem on two-trees. Technical report, Départment de Mathemátiques, EPF Lausanne, 1985. Working paper RO 850315.

[36] Darko Skorin-Karpov. On the core of the minimum cost Steiner tree game in networks. Annals of Operations Research, 57:233-249, 1995.

[37] Arie Tamir. On the core of network synthesis games. Math. Prog., 50:123-135, 1991.

[38] Arie Tamir. On the core of cost allocation games defined on location problems. Transportation Science, $27: 81-86,1993$.

[39] Richard Wong. A dual ascent approach for Steiner tree problems on a directed graph. Math. Prog., 28(3):271-287, 1984.

[40] H. Peyton Young. Cost allocation. In Robert Aumann and Sergiu Hart, editors, Handbook of Game Theory with Economic Applications, volume 2, chapter 34, pages 1194-1235. Elsevier/North-Holland Science Publishers, 1994.

\section{A Connection-Restricted Facility Location}

In a CRFL problem there is a set $T$ of terminals and a set $F$ of facilities. We set $n_{t}=|T|$ and $n_{f}=|F|$. In addition to the UFL problem each facility has a set of allowable subsets $\mathcal{A}_{f} \subseteq 2^{T}$. The goal is to open a subset of facilities and buy a set of connections of minimum total cost, such that each terminal is connected to an opened facility, and the set of terminals connected to each opened facility $f$ is in $\mathcal{A}_{f}$. In the $C R F L$ game each player owns a terminal, i.e., $K=T$. The constraint of player $t$ is satisfied if there is a bought connection $(t, f)$ to some opened facility $f$, and the subset of terminals that have a bought connections to $f$ is in $\mathcal{A}_{f}$. We can formalize the CRFL problem by an integer program as follows:

$$
\begin{array}{lll}
\text { Min } & \sum_{f \in F} c(f) y_{f}+\sum_{t \in T} c(t, f) x_{t f} & \\
\text { subject to } & \sum_{f \in F} x_{t f} \geq 1 & \text { for all } t \in T \\
& \left(y_{f}, x_{1 f}, \ldots, x_{n_{f}}\right) \in \mathcal{A}_{f} & \text { for all } f \in F \\
& y_{f}, x_{t f} \in\{0,1\} & \text { for all } t \in T, f \in F,
\end{array}
$$


where

$\mathcal{A}_{f}=\{(0, \ldots, 0)\} \cup\left\{\left(1, \chi_{A_{f}}\right) \mid A_{f} \subseteq T\right.$ feasible for $\left.f\right\} \subseteq\{0,1\}^{n_{t}+1}$, and $\chi_{A_{f}}$ denotes the characteristic vector of the subset $A_{f}$.

We here concentrate on a subclass of closed games (denoted CCRFL). In these games the sets $\mathcal{A}_{f}$ are downward closed, i.e., for every $A \subseteq A^{\prime} \in \mathcal{A}_{f}$ we have $A \in \mathcal{A}_{f}$. Note that this class encompasses a large variety of facility location problems considered in the literature, e.g., with capacity or incompatibility constraints.

Theorem 12. If a CCRFL game $\Delta$ has a non-empty core, then the strategic game $\Gamma$ has a strong equilibrium.

Proof. Following the argumentation in [18] it is possible to use the conic hull of the sets $\mathcal{A}_{f}$ to derive a linear relaxation:

$$
\begin{array}{lll}
\text { Min } & \sum_{f \in F} c(f) y_{f}+\sum_{t \in T} c(t, f) x_{t f} & \\
\text { subject to } & \sum_{f \in F} x_{t f} \geq 1 & \text { for all } t \in T \\
& \left(y_{f}, x_{1 f}, \ldots, x_{n_{t}}\right) \in \operatorname{cone}\left(\mathcal{A}_{f}\right) & \text { for all } f \in F .
\end{array}
$$

For this program a dual can be given by

$$
\begin{array}{ll}
\operatorname{Max} & \sum_{t \in T} \gamma_{t} \\
\text { subject to } & \sum_{\substack{t \in A_{f} \\
\text { for } f \in F \text { and }{ }_{f} \in \mathcal{A}_{f} .}} \gamma_{t} \leq c(f)+\sum_{\substack{t \in A_{f} \\
A_{f}}} c(t, f)
\end{array}
$$

Now we can apply similar arguments as before. An integral optimum solution $\left(x^{*}, y^{*}\right)$ to the LP-relaxation represents a partition of the terminal set $T$ into a collection of feasible sets $A_{f}^{*}$, one for each facility $f$. The constraints corresponding to these sets hold with tightness, and we can assign each player $t$ to pay for her terminal the amount $s_{t}(t, f)=\min \left\{\gamma_{t}^{*}, c(t, f)\right\}$ as connection cost to $f$ with $t$ connected to $f$, in which $\gamma^{*}$ is the optimum solution to the dual. For the opening costs $s_{t}(f)=\max \left\{\min \left\{c(f), \gamma_{t}^{*}-c(t, f)\right\}, 0\right\}$. Note that such an assignment is always possible due to $\mathcal{A}_{f}$ being downward closed. In particular, no player $t$ is required to pay for the connection cost of any other player. Thus, no coalition of players can improve by simply dropping payments.

In total this pays exactly for all costs of the solution by duality. Suppose there is a violating coalition $C$. This coalition must be able to connect their terminals differently at a cheaper total cost. Consider the strategy vector after the coalition has changed its strategy. Each member $t^{\prime} \in C$ must again be part of some $A_{f^{\prime}}$ for some facility $f^{\prime}$, for which the total (connection + opening) costs are fully paid for. In particular, the new payments exactly pay for $c\left(f^{\prime}\right)+\sum_{t \in A_{f^{\prime}}} c\left(t, f^{\prime}\right)$. Note that no player has increased his payments, but $t^{\prime}$ has strictly decreased his payments. This means that the original payments coming from $\gamma_{t}^{*}$ violate the dual constraint corresponding to $\mathcal{A}_{f^{\prime}}$. This is a contradiction to $\gamma^{*}$ being the optimal dual solution.

The recognition of SE can be done similarly as for UFL games.

Corollary 8. Given a strategy vector for a CCRFL game $\Gamma$ we can verify in polynomial time if it is a SE. 